\title{
How consistent are drivers in their driving? A driver training perspective
}

\author{
H. Malik, A. Rakotonirainy, G. Larue \& F. Maire \\ Center of Accident Research and Road Safety, Queensland, Australia
}

\begin{abstract}
The value and effectiveness of driver training as a means of improving driver behaviour and road safety continues to fuel research and societal debates. Knowledge about what are the characteristics of safe driving that need to be learnt is extensive. Research has shown that young drivers are over represented in crash statistics. The encouraging fact is that novice drivers have shown improvement in road scanning pattern after training. This paper presents a driver behaviour study conducted on a closed circuit track. A group of experienced and novice drivers performed repeated multiple manoeuvres (i.e. turn, overtake and lane change) under identical conditions Variables related to the driver, vehicle and environment were recorded in a research vehicle equipped with multiple invehicle sensors such as GPS accelerometers, vision processing, eye tracker and laser scanner. Each group exhibited consistently a set of driving pattern characterising a particular group. Behaviour such as the indicator usage before lane change, following distance while performing a manoeuvre were among the consistent observed behaviour differentiating novice from experienced drivers. This paper will highlight the results of our study and emphasize the need for effective driver training programs focusing on young and novice drivers.

Keywords: driver training, overtake, turn, novice and experienced drivers.
\end{abstract}

\section{Introduction}

Road crashes are the single highest killer of 15-24 year-olds in industrial countries [1]. Driving is a complex task which requires the driver to assess subjectively their position with respect to the lanes and surrounding vehicles and anticipate the future trajectory of their vehicle within that scenario. Therefore driving safely takes time to learn and needs extensive practice. With time, the 
actions of driving i.e. changing gears, looking in the rear-view mirror, steering, correctly assessing situations, reacting appropriately, etc. becomes a naturalistic behaviour and efficient. However, the novice driver has to think about these actions, increasing overall mental workload and possibly distracting attention from the road [2]. It has been demonstrated that a major contributing factor to crashes of newly licensed driver, is the failure to scan effectively for potential risks [3-6]. We hypothesize that the failure to understand what is really important for inexperienced driver to learn, in terms of risky driving, is one of the many reasons restraining us to build better training programs.

Relatively little research has focused on the different errors that drivers make, or about the causal factors that contribute to these errors made by drivers [7]. Inorder to comprehensively tackle driving issues, a complete and integrated framework needs to be developed that should include and examine all the parameters that influence driving (i.e. cues related to road, vehicle and driver). Our study comprise of assessing the consistency of the driving behaviour during manoeuvres, based on the parameters acquired from the Driver, Vehicle and Environment (DVE). Once the assessment has been made, an effective feedback system needs to be put in place that can help driver trainers to better explain the driving drawbacks of novice trainee drivers.

This paper describes the driving experiment in detail that was designed with the help of driver trainers to monitor the consistency of the driver behaviour. It helped to also understand the different behaviours demonstrated by the observed groups of drivers (i.e. novice and experienced). This paper also discusses the results in the form of relationship between the multiple driving variables recorded in terms of experience. GLMs (Generalised Linear Models) [8] were used to identify the driving variables that are influenced by experience.

\section{The driving experiment}

The objective of this study was to observe and assess driving competencies for both novice and experienced drivers using a 4WD. For this project, a driver training organisation (known as 'Roadcraft'), Queensland agreed to provide the track for conducting the test drives. Figures 1 and 2 shows the test track used for this experiment. The authors will like to acknowledge the assistance of MURCOTTS Pty Ltd. and its employees in selecting the driving manoeuvres and test track for the driving experiment. Detailed specifications of the study are presented below.

\subsection{Vehicle specifications}

A Toyota 4WD was used as the test vehicle which the participants drove during the driving experiment. This vehicle was provided by QFleet and was equipped with the multiple sensors for evaluating the driving parameters. A second vehicle was used in overtaking manoeuvres. Specifications for the two vehicles used during the driving experiment are provided here. 


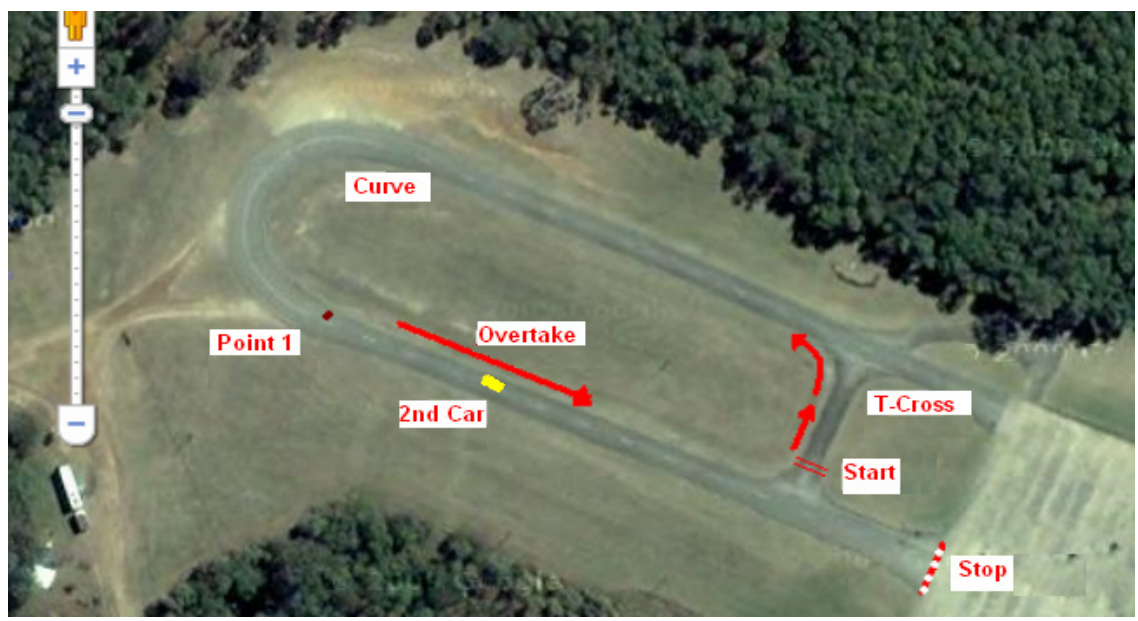

Figure 1: $\quad$ First loop of each driving scenario (first lap).

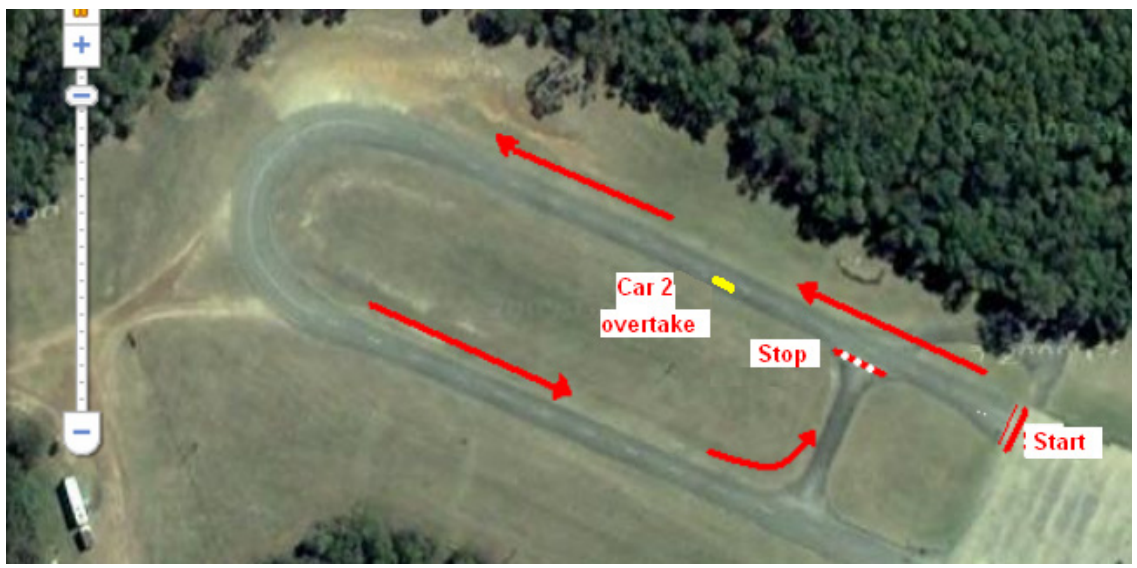

Figure 2: $\quad$ Second loop of each driving scenario (second lap).

A 4WD vehicle (test vehicle) that contained the necessary sensors and laptops for monitoring the driving performance. The sensors included

- MobileEye and IBEO Laser scanner for lane and obstacle detection

- GPS and Vigil system for vehicle position and vehicle dynamics

- faceLAB for tracking drivers head/eye movements

- Cameras to record the images of the road ahead for gaze evaluation

A second vehicle (Toyota hatchback) was provided by Roadcraft and driven by RoadCraft's driver. This vehicle was used in the overtake manoeuvres.

Other than these two mentioned vehicles, there were no other vehicles on the test track. 


\subsection{Test track specifications}

The driving experiment was conducted on a closed loop track. The pictorial representation of the track is presented in figures 1 and 2. Specifications for the test track/circuit are given below

- A fixed start point and end point from where the test vehicle starts and stops respectively.

- Clearly visible marked lanes (two lanes) for the execution of the overtake manoeuvres. The straight stretch of the track was approximately 150 metres long for execution of overtake manoeuvre at $50 \mathrm{~km} / \mathrm{h}$. (maximum limit). The vehicle being overtaken was set to have a maximum speed of $25 \mathrm{~km} / \mathrm{h}$.

- Clearly visible marked lanes for manoeuvring a left turn on T-junction.

- Clearly visible marked lanes for a road curve/turn manoeuvres.

\subsection{Participants}

Three experienced drivers (i.e. driver trainers) were selected from the driver training school (Roadcraft). The experienced drivers had an average of 20 years of driving experience. The inexperienced drivers (i.e. novice drivers) were recruited from the locality of Gympie, Queensland. The novice drivers were three male participants, each having a driving licence for less than two years and less than one year of on-road driving experience. All drivers had a valid driving license. Along with this, one driver trainer was recruited for the vehicle that was used for overtaking manoeuvres. Another driver trainer was recruited to monitor the participants and provide subjective assessments.

Each participant was given two test laps to allow them to become familiar with the track and start/stop locations. The setting up of sensors and profile creation for each participant was finalised before the test laps. All subjects provided written consent for this study which was approved by QUT ethics committee. Participants were paid AUD \$50 for completing the driving sessions.

\subsection{Procedure}

The participants drove a Toyota 2007 Land Cruiser (i.e. 4WD). The track used for the test drive is presented in figures 1 and 2. The drivers' eye/head movement along with vehicle dynamics and lane/obstacle positioning was recorded. Each driving task was divided into two loops. Each loop started and stopped at the positions identified in figures 1 and 2. The maximum speed limit for driving on this track was set to $50 \mathrm{~km} / \mathrm{h}$.

Before the start of the experiment, each drivers' face model was created using faceLAB for eye/head tracking. Furthermore, drivers' details such as age, driving experience (in number of years), gender, were recorded. The information collected was treated in a confidential manner. Each driver was also briefed about the track geometry and the driving manoeuvres they would have to perform. 


\section{- $\quad$ First lap}

In the first lap, drivers were instructed to turn left at the T-crossing. After going through the 'curve', the drivers overtook a vehicle that was in position (as shown in Figure 1 '2nd car'). "Point 1" is a reference point for the driver in the "2nd car". The moment the $4 \mathrm{WD} /$ test vehicle reached this reference point, the driver of the "2nd Car" started to drive and did not drive at more than $25 \mathrm{~km} / \mathrm{h}$. The driver of the 4WD after overtaking the '2nd Car' stopped at the point shown in Figure 1. After completely stopping at the designated point (see Figure 1), the driver of the $4 \mathrm{WD} /$ test vehicle positioned the car for the second loop of the driving scenario (see 'Start' position in Figure 2).

\section{- Second lap}

The start point of the second loop is pointed out in Figure 2. In this loop the driver once again overtook the "Car 2" that was travelling at no more than $25 \mathrm{~km} / \mathrm{h}$. After the curve of the track, the $4 \mathrm{WD} /$ test vehicle drivers made a left turn and stopped at the designated position as pointed out in Figure 2. Each driver had to complete all laps (i.e. two loops) and the selected manoeuvres ten times.

\section{- Data collection}

Data was collected at a high frequency (varying frequencies (ranging from $1 \mathrm{~Hz}-$ $55 \mathrm{~Hz}$ ) of sensory data obtained from the car, the environment and the driver). Data from GPS was retrieved at $1 \mathrm{~Hz}$, vehicle dynamics and lane/obstacle monitoring system at $5 \mathrm{~Hz}$, driver's head and eye gaze data at $55 \mathrm{~Hz}$, cameras at $30 \mathrm{~Hz}$, laser scanner at $50 \mathrm{~Hz}$. The data collected from the three components of driving is given below in Table 1 .

Table 1: Data (driving variables) collected during the experiment / study.

\begin{tabular}{ccc}
\hline Vehicle & Driver & Environment \\
\hline \hline Average Speed & Head position & Lane position of the test vehicle \\
Brake & Gaze position & Obstacle position in-front of test vehicle \\
Indicator state & & Obstacle position behind the test vehicle \\
Excess & & GPS location \\
acceleration & & \\
Excess & & \\
deceleration & & \\
\hline
\end{tabular}

\section{Results}

As already mentioned in the section above that multiple manoeuvres were chosen by the driver trainers to be monitored, but in this paper the results of overtake and turn will be discussed. This section will objectively identify the 
recorded driving variables (that are mentioned in Table 1) that are influenced with the change in driving experience.

To quantify this difference in multiple driving behaviours (i.e. gaze pattern, speed, lane keeping) between novice and experienced drivers, Generalized Linear Models (GLMs) were used. GLMs from the Poisson family were fitted to obtain the expected value of influenced factor (i.e. driving experience) knowing the influencing/following factors: manoeuvre and position of the gaze on the region of the road ahead, speed, lane distance etc.

The formula below models an example relationship between gaze frequency and the experience mentioned above.

$$
\begin{cases}\eta=\sum_{i} \beta_{i} \cdot \text { Factor }_{i} & \rightarrow(a) \\ E(\text { No. of gaze } \mid \text { Factors })=\exp (\eta) & \rightarrow(b)\end{cases}
$$

where Factor $_{i}$ is either 1 or 0 and $\beta$ is the estimate for the factors (refer to Table 1). In order to compute the expected number of gaze in a region/segment, (a) returns $\eta$ (eta) which is a linear combination of the factors that we want to investigate. The link function logarithm is used to model the relationship between the linear predictor (eta) and the expected number of gaze given the factors (i.e. experience). This is presented in (b) using the inverse link function (exp).

The impacts of the different factors obtained using GLM for evaluating gaze span are summarized in Table 1 below. All these factors are statistically significant ( $p$-value $<0.05$ ). The level of statistical significance as assessed by $p$ value is represented by the number of ' $*$ '.

Table 2 and 3 provide empirical differences of the driving variables (i.e. gaze span, speed etc) between novice and experienced drivers as they performed the turn manoeuvres.

Table 2 helps to understand the frequency of the gaze points by the two groups of drivers namely novice and experienced. The frequency was monitored for a certain portion of the road ahead of the driver. It can be seen from the table 2 that the frequency of the gaze within the monitored section (i.e. straight ahead) was higher for novice as compared to experienced drivers. Novice drivers showed a narrow gaze span by monitoring just the road straight ahead as compared to experienced drivers who monitored a wider area on the road ahead.

Table 2: $\quad$ Linear regression estimates for factors influencing gaze span.

\begin{tabular}{|c|c|c|c|c|}
\hline Factors & Estimate $(\beta)$ & Std. Error & p-value & Code \\
\hline Intercept $(\alpha)$ & 5.078732 & 0.008475 & $<2 \mathrm{e}-16$ & $* * *$ \\
\hline Inexperienced & 0.416702 & 0.007355 & $<2 \mathrm{e}-16$ & "*** \\
\hline $\begin{array}{l}\text { Signif. codes: } \\
\text { p-val }=0 \text { ‘***, }\end{array}$ & p-val $<0.001^{\prime} * *$, & p-val $<0.01^{\prime *} *$ & p-val $<0.05$ ' & \\
\hline
\end{tabular}


The other factor that was observed to vary with experience was the speed during the turn. It can be observed from table 3 that the inexperienced/novice drivers had lower speed as they performed the turn manoeuvres.

Table 3: Linear regression estimates for factors influencing speed through the turn.

\begin{tabular}{ccccc}
\hline \hline Factors & Estimate $(\beta)$ & Std. Error & p-value & Code \\
\hline Intercept $(\boldsymbol{\alpha})$ & 33.0054 & 0.3876 & $<2 \mathrm{e}-16$ & $* * *$ \\
\hline \hline Inexperienced & -2.0557 & 0.4650 & $<2 \mathrm{e}-16$ & $* * *$ \\
\hline $\begin{array}{l}\text { Signif. codes: } \\
\text { p-val }=0 \text { 0***, }\end{array}$ & p-val $<0.001^{* * *}$, & $\mathrm{p}-\mathrm{val}<0.01^{\circ *}$, & $\mathrm{p}$-val $<0.055^{*}$, & \\
\hline \hline
\end{tabular}

Now this may not be a less competent manner of driving, but it was an observation that differentiated the novice from experienced drivers during the driving experiment.

The other manoeuvre under consideration in this paper is the overtake manoeuvre. Tables 4 and 5 provide the results of the factors that were influenced by the experience of the drivers during the performed overtake manoeuvres.

Table 4: Regression estimates for factors influencing 'distance of the test vehicle from the right lane' during the overtake manoeuvres.

\begin{tabular}{ccccc}
\hline \hline Factors & Estimate $(\beta)$ & Std. Error & p-value & Code \\
\hline Intercept $(\alpha)$ & 96.906 & 1.883 & $<2 \mathrm{e}-16$ & $* * *$ \\
\hline \hline Inexperienced & 23.356 & 2.837 & $<2 \mathrm{e}-16$ & $* * *$ \\
\hline $\begin{array}{l}\text { Signif. codes: } \\
\text { p-val=0 } 0^{\text {‘***, }}\end{array}$ & p-val $<0.001^{\text {‘**, }}$ & $\mathrm{p}$-val $<0.01^{\text {‘*}}$, & $\mathrm{p}$-val $<0.05 *$, & \\
\hline \hline
\end{tabular}

Table 5: Regression estimates for factors influencing 'amount of time spent in the right lane' during the overtake manoeuvres.

\begin{tabular}{ccccc}
\hline \hline Factors & Estimate $(\beta)$ & Std. Error & p-value & Code \\
\hline Intercept $(\boldsymbol{\alpha})$ & 7.5375 & 0.1305 & $<2 \mathrm{e}-16$ & $* * *$ \\
\hline \hline Inexperienced & -0.7203 & 0.1871 & $<2 \mathrm{e}-16$ & $* * *$ \\
\hline $\begin{array}{l}\text { Signif. codes: } \\
\text { p-val=0;***, }\end{array}$ & $\mathrm{p}$-val $<0.001^{\text {‘** }}$, & $\mathrm{p}$-val $<0.01^{\text {‘* }}$, & $\mathrm{p}$-val $<0.055^{*}$, & \\
\hline \hline
\end{tabular}

It should be noted that the experiment was conducted in a region where the vehicles are right hand drives therefore the distance of the vehicle was also considered from the right hand lane. Table 4 shows the relationship between the distance of the test vehicle from the right lane (in $\mathrm{cms}$ ) and other driving factors (i.e. experience) monitored during the overtake manoeuvre. A significant difference was observed for this driving variable (i.e. distance of the test vehicle 
from the right lane) in relation with driving experience. Novice drivers tend to pass the overtaken vehicle giving a very small margin on their left side. Whereas, the experienced drivers gave much more distance to the car on the left which they overtook.

Another significant observation was related to the time duration spent in the right lane as the two observed groups of drivers overtook a vehicle. This observed factor that distinguished the novice and experienced drivers during the analysis of the overtake manoeuvres is presented in Table 5. It shows that the time spent in the right lane whilst overtaking is less for inexperienced/novice drivers. This demonstrates that as novice drivers are passing the vehicle to be overtaken using the right lane; they tend to stay in the right lane for a shorter amount of time as compared to their experienced counterparts.

\section{Conclusion}

Young drivers have a high crash liability relative to those with a few years of driving experience. This suggests that experience teaches some skills that enable experienced drivers to avoid crashes. By teaching such skills to young drivers through effective driver training would adversely impact young driver crash rates.

Consistency in a number of driving variables has been found between novice and experienced drivers through the driving experiment presented in this paper. This also helped to identify the behaviours that the different groups of drivers demonstrate as they performed the selected driving manoeuvres. The experiment depicted that novice drivers have a shorter gaze span as compared to their experienced counterparts. In addition, novice drivers' tend to have low speed during the turn manoeuvres. For the overtake manoeuvres, novice drivers are inclined to give less distance on the left side to the overtaken vehicle. Along with this, it was observed that during the overtake manoeuvre, novice drivers spend less time in the right lane as compared to experienced drivers.

These observations can be used in future to design a rule based driving model which has clear practical applications both in testing and training of drivers. Such a model would allow driving attributes to be identified that are required for highly competent manoeuvring. The rules designed will enable driver trainers to assess drivers' behaviour as high/low competent. Feedback on the attempted driving manoeuvres, highlighting both strengths and weaknesses of the driver will act as an effective measure to improve driving skills particularly amongst novice drivers.

\section{References}

[1] Young Drivers: The Road to Safety, Policy Brief, Organisation For Economic Co-Operation And Development, October 2006.

[2] CLARKE, D., FORYST, R and WRIGHT, R., "A statistical profile of road accidents during cross-flow turns", Accident Analysis and Prevention, 37, 721-730.2005. 
[3] MCKNIGHT JA, MCKNIGHT SA. Young novice drivers: Careless or clueless. Accident Analysis Prevention, 35:921-5. 2003.

[4] TREAT JR, TUMBAS NS, et al. Tri-level study of the causes of traffic accidents (Final Report). Causal factor tabulations and assessments, vol I. Bloomington, IN: Indiana University Institute for Research in Public Safety, 1979.

[5] FISHER DL, POLLATSEK A. Novice driver crashes: failure to divide attention or failure to recognize risks. Kramer AF, Kirlik A, Wiegmann D (eds). Attention: from theory to practice. Oxford: Oxford University Press (in press).

[6] PRADHAN A, HAMMEL KR, et al. Using eye movements to evaluate effects of driver age on risk perception in a driving simulator. Human Factors, 47:840-52. 2005.

[7] NEVILLE A. Stanton and PAUL M. Salmon, Driver error and the implications for intelligent transport systems design, 16th World Congress on Intelligent Transportation Systems, Stockholm, Sweden. 2009.

[8] NELDER, J. and WEDDERBURN, R. Generalized linear models. Journal of the Royal Statistical Society. 135 (3), 370-384, 1972. 
